\title{
Passivating ligand and solvent contributions to the electronic properties of semiconductor nanocrystals $\dagger$
}

\author{
Sean A. Fischer, ${ }^{a}$ Angela M. Crotty, ${ }^{c}$ Svetlana V. Kilina, ${ }^{b}$ Sergei A. Ivanov ${ }^{c}$ and Sergei Tretiak ${ }^{* c}$ \\ Received 26th September 2011, Accepted 17th November 2011 \\ DOI: $10.1039 / \mathrm{c} 2 \mathrm{nr} 11398 \mathrm{~h}$
}

\begin{abstract}
We examine in detail the impact of passivating ligands (i.e., amines, phosphines, phosphine oxides and pyridines) on the electronic and optical spectra of $\mathrm{Cd}_{33} \mathrm{Se}_{33}$ quantum dots (QDs) using density functional theory (DFT) and time-dependent DFT (TDDFT) quantum-chemical methodologies. Most ligand orbitals are found deep inside in the valence and conduction bands of the QD, with pyridine being an exception by introducing new states close to the conduction band edge. Importantly, all ligands contribute states which are highly delocalized over both the QD surface and ligands, forming hybridized orbitals rather than ligand-localized trap states. In contrast, the states close to the band gap are delocalized over the QD atoms only and define the lower energy absorption spectra. The random detachment of one of ligands from the QD surface results in the appearance of a highly localized unoccupied state inside the energy gap of the QD. Such changes in the electronic structure are correlated with the respective QD-ligand binding energy and steric ligand-ligand interactions. Polar solvent significantly reduces both effects leading to delocalization and stabilization of the surface states. Thus, trap and surface states are substantially eliminated by the solvent. Polar solvent also blue-shifts (e.g., $0.3-0.4 \mathrm{eV}$ in acetonitrile) the calculated absorption spectra. This shift increases with an increase of the dielectric constant of the solvent. We also found that the approximate single-particle KohnSham (KS) approach is adequate for calculating the absorption spectra of the ligated QDs. Besides a systematic blue-shift, the KS spectra are in very good agreement with their respective counterparts calculated with the more accurate TDDFT method.
\end{abstract}

\section{Introduction}

Semiconductor nanocrystals are a class of materials with unique, size-dependent chemophysical properties. ${ }^{1-3}$ They have been studied extensively for many years for potential applications ranging from fluorescent biotags ${ }^{4}$ to quantum computing, ${ }^{5}$ from lasing ${ }^{6}$ to photovolatics. ${ }^{7}$ Much of this research has been focused on colloidal quantum dots (QDs). Advances in synthetic techniques have allowed for the creation of high quality, monodispersed samples of QDs. ${ }^{8-10}$ These high-quality samples afford an unprecedented level of experimental characterization of the electronic and optical properties of the QDs. While much is known about the size-dependence of the QD properties, ${ }^{11-13}$ considerably less is understood about the effects the surface morphology and passivation layer have. Experiments have

${ }^{a}$ Department of Chemistry, University of Washington, Seattle, WA, 98102 ${ }^{b}$ Department of Chemistry and Biochemistry, North Dakota State University, Fargo, ND, 58108. E-mail: skilina@lanl.gov

'Theoretical Division, Center for Nonlinear Studies (CNLS) and Center for Integrated Nanotechnologies (CINT), Los Alamos National Laboratory, Los Alamos, NM, 87545. E-mail:serg@lanl.gov

$\uparrow$ Electronic supplementary information (ESI) available. See DOI: $10.1039 / \mathrm{c} 2 \mathrm{nr} 11398 \mathrm{~h}$ shown that changes to the capping ligands can have a dramatic effect on the inter- and intraband charge carrier dynamics. ${ }^{6,14-17}$ For example, the intraband relaxation rate in CdSe QDs can be slowed by two orders of magnitude by changing the capping ligands. ${ }^{14}$ Radiative dynamics can also be significantly influenced by changes to the passivation layer. Using primary alkylamines, ${ }^{15}$ or overlayers of a wide band gap semiconductor, ${ }^{18}$ to passivate the surface of CdSe QDs results in substantial increases in the emission quantum yield. Surface chemistry also influences multiple exciton generation efficiencies in QDs. ${ }^{19}$

Thus achieving the desired functionality in these systems requires detailed knowledge of, and the ability to control, the QDligand interactions. Experimentally this task is difficult since conventional spectroscopic techniques cannot directly probe these interactions: electronic transitions associated with QDligand interactions are usually optically forbidden. Acquiring this knowledge theoretically is also challenging. The QDs typically used in experiments are comprised of 100 s to 1000 s of atoms, and this does not even include the ligand molecules. Semiempirical methods, such as tight-binding ${ }^{20}$ and pseudopotential ${ }^{21,22}$ approaches, have been applied to these systems. Any realistic model, however, has to explicitly describe bonding between the QD and the ligand molecules, which is lacking or not complete in 
these approaches. Several efforts have been made to model ligated CdSe QDs using force field ${ }^{23,24}$ and Monte Carlo methods, ${ }^{25}$ however, the parameterization present in such methods needs to be verified for each system and material. First-principle methods, like density functional theory (DFT), are capable of providing reasonably accurate descriptions of the interactions between QD and ligand without the starting assumptions of the semiempirical methods, yet computational tractability can quickly become an issue when discussing simulations of QDs. To overcome this, many past studies employing DFT artificially terminated unpassivated surface valences, or dangling bonds, with hydrogen atoms. $^{26-28}$ The accuracy of this passivation method has been called into question for all but a few QD materials. ${ }^{29}$

Because of significant computational cost, most DFT calculations of structural and optical properties of QDs have been performed on small clusters of a few atoms in size, ${ }^{30-34}$ usually utilizing time dependent DFT (TDDFT) for obtaining absorption spectra. Several efforts have been made to address the specifics of the ground state electronic structure ${ }^{35-37}$ and binding energies of larger CdSe clusters (1.3-2 $\mathrm{nm}$ in diameter) interacting with a single ligand molecule ${ }^{38,39}$ and with many ligands fully or partially passivating the surface of the CdSe QD. ${ }^{40,41}$ However, these studies either do not consider optical properties at all, or have utilized a single-particle, mean-field representation of optical transitions. ${ }^{40}$ Such an approach can be used as a zeroth-oder approximation for calculations of absorption spectra of inorganic nanomaterials, where the confinement energy is much larger than the Coulomb interaction between a photoexcited electron-hole pair. ${ }^{2}$ However, this approach is expected to fail in the case of organic molecules typically exhibiting strong electronic correlations. As such, applicability of the single-particle approach to simulate optical response of QDs passivated with organic ligands is not clear. Additionally, the majority of theoretical studies concerning semiconducting nanocrystals have been done in the gas-phase, while the majority of experimental measurements have been done in the solution phase. There have been a few recent papers ${ }^{42-44}$ looking to address the role of solvent on the properties of these materials; however, the small sizes of the systems used in these studies $(<20$ atoms), raises concern over whether the conclusions drawn are applicable to the nanocrystals typically used in experiments.

In the present work, we continue our previous studies ${ }^{40}$ involving $\mathrm{Cd}_{33} \mathrm{Se}_{33}$ QDs capped with realistic model ligands. CdSe QDs of comparable diameter are experimentally accessible, ${ }^{45,46}$ and their size and shape are controllable. ${ }^{47}$ Recently, DFT calculations have been undertaken on larger QD systems; ${ }^{48,49}$ however, the additional computational cost of these larger systems makes comprehensive studies, like the present work, unreasonable. Four different ligands fully passivating the surface of the $\mathrm{Cd}_{33} \mathrm{Se}_{33}$ QD have been considered. These molecules represent models of commonly used ligands with colloidal QDs: methylamine $\left(\mathrm{NH}_{2} \mathrm{Me}\right)$, trimethylphosphine $\left(\mathrm{PMe}_{3}\right)$, trimethylphosphine oxide $\left(\mathrm{OPMe}_{3}\right)$, and pyridine (see Fig. 1). We have focused on the questions of whether passivating ligands introduce trap states inside or near the energy band gap of the QD and what the effect losing a ligand has on the electronic structure and absorption spectra of QDs. The role of the solvent on the optoelectronic properties of passivated QDs has been investigated, as well. We also examine the validity of absorption spectra calculated within the single-particle Kohn-Sham (KS) method $^{40}$ by comparing to those obtained with the linear response TDDFT approach that incorporates many-body correlation effects including Coulombic interactions between photoexcited electrons and holes.

The paper is organized as follows: our computational methodology is outlined in section 2. In section 3 we present the results of our simulation of the properties of the QD-ligand systems and the validity of the KS spectra. We conclude by summarizing our results and observed trends in section 4.

\section{Methodology}

The starting point for the geometries was a roughly spherical CdSe cluster cut from the bulk lattice. Previous computational $^{30,39}$ and experimental studies ${ }^{16,50}$ indicate that ligands bind preferentially to the surface $\mathrm{Cd}$ atoms. Therefore, 21 ligands of each type (see Fig. 1) were initially attached to $\mathrm{Cd}$ atoms on the surface of the $\mathrm{Cd}_{33} \mathrm{Se}_{33}$ structure. Although various acidic phosphorus species have been recently discovered to play an important role in passivating the surface of colloidal QDs, ${ }^{51-54}$ here we consider only neutral ligands (methylamine, trimethylphosphine, trimethylphosphine oxide, and pyridine) that do not provide additional charge(s) to the QD.

The geometry optimizations were performed with the Gaussian-03 software package, ${ }^{55}$ using DFT at the B3LYP/ LANL2dz (functional/basis set) level of theory. Electronic structure and optical response calculations on the optimized geometries were performed with Gaussian-0956 and Gaussian-03 using the B3LYP functional with the LANL2dz/6-31G* basis set, where the relativistically-corrected Effective Core Potential (EPS), double-zeta quality LANL2dz basis set has been used for the QD atoms ( $\mathrm{Cd}$ and $\mathrm{Se}$ ) and the 6-31G* basis set has been chosen for ligand atoms $(\mathrm{C}, \mathrm{H}, \mathrm{N}, \mathrm{O}$, and $\mathrm{P})$. Our recent investigations ${ }^{57}$ have demonstrated that the combination of B3LYP functional and LANL2dz/6-31G* basis set is effective in providing an accurate description of ligand-QD interactions. To characterize the strength of the ligand-QD interactions, we calculate binding energies as

$$
E_{B}=E_{Q D+21 L i g}-\left(E_{Q D+20 L i g}+E_{L i g}\right)
$$

where $E_{B}$ is the binding energy, $E_{Q D+21 \text { Lig }}$ is the total energy of the optimized $\mathrm{Cd}_{33} \mathrm{Se}_{33} \mathrm{QD}$, whose surface is fully passivated by 21 ligands, $E_{Q D+20 \text { Lig }}$ is the energy of the ligated QD missing one ligand, and $E_{L i g}$ is the energy of an isolated ligand. The geometry of the system was left unchanged following removal of the ligand. Negative values of $E_{B}$ define binding between the QD and ligands: the more negative the value of the binding energy, the stronger the QD-ligand interaction. If $E_{B} \geq 0$, the QD-ligand interaction is repulsive, and ligands may detach from the QD surface after overcoming the respective bond-breaking barrier.

Optical absorption spectra and the density of states (DOS) were calculated within the single-particle (Kohn-Sham representation) and linear response TDDFT technique, utilizing Gaussian line-broadening:

$$
A(\omega)=\frac{1}{\sigma \sqrt{(2 \pi)}} \sum_{n} h_{n} \exp \frac{-\left(\omega_{n}-\omega\right)^{2}}{\sigma^{2}}
$$



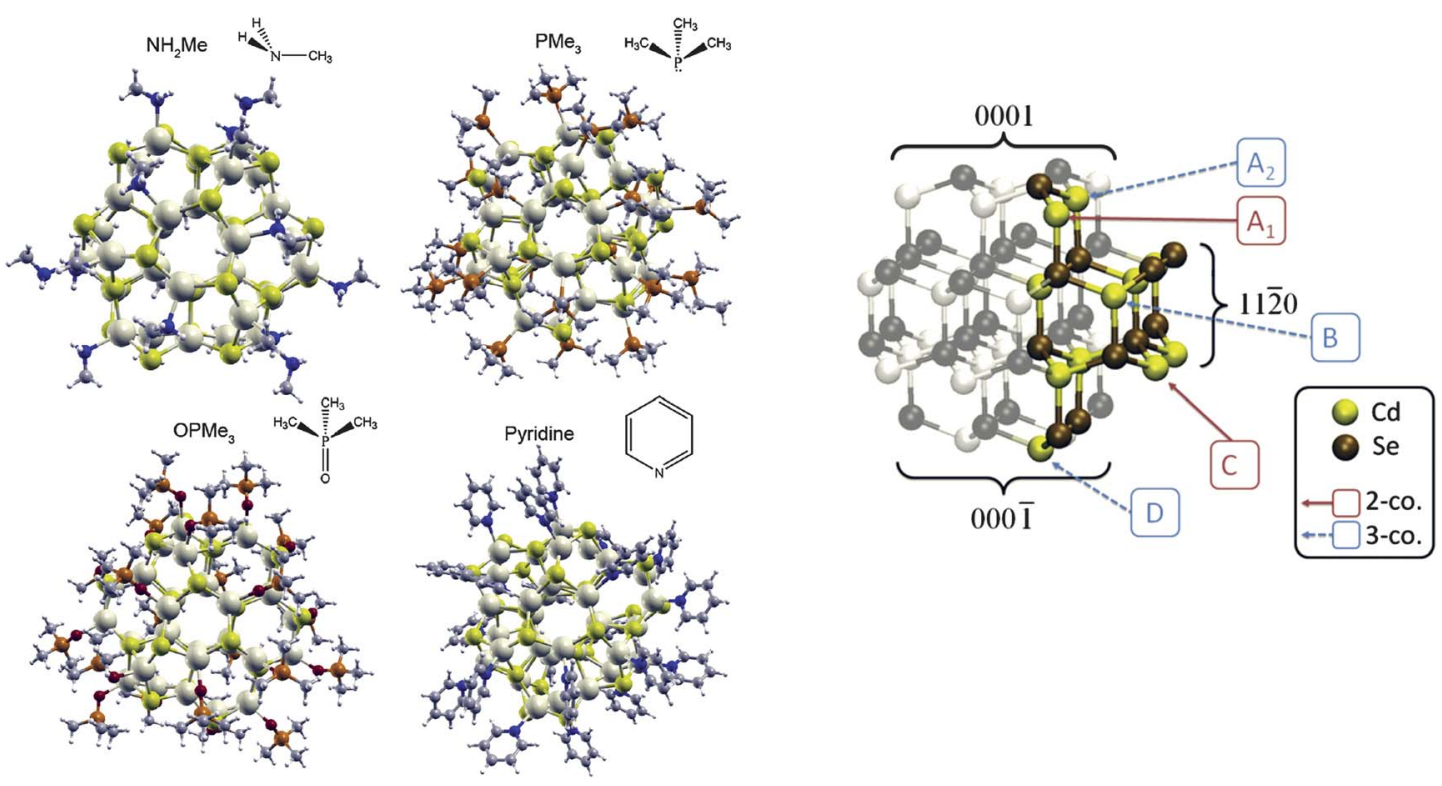

Fig. 1 Optimized geometries of the four ligated QD clusters we study. The ligands used are methylamine $\left(\mathrm{NH}_{2} \mathrm{Me}\right)$, trimethylphosphine $(\mathrm{PMe})_{3}$, trimethylphosphine oxide $\left(\mathrm{OPMe}_{3}\right)$, and pyridine, as shown on the insets. The right-hand panel shows the initial Cd ${ }_{33} \mathrm{Se}_{33}$ configuration obtained from bulk CdSe crystal. One-third of the crystal is in color, highlighting its three-fold symmetry. 2- and 3-coordinated atoms on the surface of the cluster are listed in red and blue, respectively. Miller-Bravais indices for surfaces of the cluster are given in black. Geometry optimization of the cluster leads to a reconstruction of the surface so that all surface atoms become at least 3-coordinated.

where $\omega_{n}$ is the energy of transition, $h_{n}$ is the 'strength' of transition (see below), and $\sigma$ is an empirical line-broadening parameter. For the DOS plots, $\omega_{n}=\varepsilon_{n}\left(\varepsilon_{n}\right.$ being the $n$th molecular orbital (MO) energy, and the index $n$ runs over occupied and virtual MOs) and $\sigma=100 \mathrm{meV}$. For the total DOS plots, $h_{n}=1$, while for the fragment decomposition of the DOS, $h_{n}$ is the fraction of the respective molecular orbital localized on the specified fragment, as incorporated in the Gaussian-09 code. For the absorption spectra, $\omega_{n} \equiv \omega_{k l}=\varepsilon_{k}-\varepsilon_{l}\left(\varepsilon_{k}\right.$ and $\varepsilon_{l}$ being the energies of the virtual and occupied MOs, respectively, and the indices $k$ and $l$ run over the occupied and virtual orbital space, respectively), $h_{n} \equiv h_{k l}=f_{k l}\left(f_{k l}\right.$ is the oscillator strength of the respective transition between $k$ th and $l$ th MOs), and $\sigma=30 \mathrm{meV}$. Finally, the oscillator strength is given as

$$
f_{k l}=\frac{2 m_{e}\left(\varepsilon_{k}-\varepsilon_{l}\right)}{3 \hbar^{2}} \mu_{k l}^{2}
$$

where $\mu_{k l}=\left\langle\psi_{k}|\hat{\mu}| \psi_{l}\right\rangle$ is the transition dipole moment, $\psi_{k}$ and $\psi_{l}$ are the respective MO wavefunctions and $\hat{\mu}$ is the dipole moment operator. The absorption spectra calculated within the KS approach are compared with those calculated using linear response TDDFT as implemented in the Gaussian code. The latter computations provided the respective transition energies $\omega_{n}$ and oscillator strengths $h_{n}=f_{n}$ for eqn (2). The same basis set and functional were utilized for both methods. The first 50 singlet transitions were considered, and Gaussian spectral line broadening with a $30 \mathrm{meV}$ width was used for broadening of the calculated oscillator strengths to produce the spectra. Note, all KS absorption spectra and densities of transitions (DOT) have been calculated within the $0-4 \mathrm{eV}$ energy window, whereas the TDDFT absorption spectra are given only in the energy range spanned by the lowest 50 singlet transitions.
In addition to the gas-phase calculations, the electronic structure and absorption spectra have been also calculated in the solvent acetonitrile $\left(\mathrm{CH}_{3} \mathrm{CN}\right)$, which is frequently used in the relevant experimental studies. ${ }^{58-60}$ Solvent effects were simulated by embedding the system in a polarizable continuum medium with an appropriate dielectric constant $(\varepsilon=35.688$, acetonitrile) in the framework of the polarizable continuum model (PCM) ${ }^{61}$ For consistency, when solvent calculations were done with Gaussian-09, the Gaussian-03 defaults were utilized, unless otherwise noted.

\section{Results and discussion}

\subsection{Binding of ligands to the CdSe surface}

First, we consider fully passivated structures (i.e. one ligand coordinated to every two- or three-coordinated surface $\mathrm{Cd}$ atom) with a total of 21 ligands attached to $\mathrm{Cd}_{33} \mathrm{Se}_{33}$ cluster, as illustrated in Fig. 1. Even with passivating ligands, there is significant reconstruction of the QD surface. This is corroborated by recent resonant Raman studies on CdSe nanocrystals where a downshift and broadening of the longitudinal optical phonon band relative to the bulk was attributed to bond distortion. ${ }^{46,62}$ The binding energies characterizing the strength of the interaction between the QD surface and different ligands in the gas-phase are given in Table 1, where a negative number indicates favorable binding, as follows from eqn (1). Table 1 also compares our results with previously reported calculations of the binding energies between CdSe and typical ligand molecules. ${ }^{34,38-41}$ The obtained values are comparable, but larger (in absolute values) than those reported for ligands bound to the bulk CdSe surfaces. ${ }^{39}$ There are several reasons for this discrepancy. First, 
Table 1 Binding energies, $E_{B}$, calculated for various ligands using eqn (1). Our calculations are compared with the results of ref. 39 obtained for bulk CdSe surface calculated by the PW91 functional, the results of ref. 38 calculated for $\left(^{a}\right) \mathrm{Cd}_{15} \mathrm{Se}_{15}$ and $\left(^{b}\right) \mathrm{Cd}_{33} \mathrm{Se}_{33}$ interacting with a single ligand using the LDA functional, the results of ref. 40 obtained for $\mathrm{Cd}_{33} \mathrm{Se}_{33}$ fully passivated with 21 ligands utilizing the PW91 functional, the results of ref. 41 for $\mathrm{Cd}_{33} \mathrm{Se}_{33}$ with $21\left(\mathrm{NH}_{3}\right)$ or $9\left(\mathrm{NH}_{2} \mathrm{Me}\right)$ ligands using $\left({ }^{c}\right)$ LDA or $\left({ }^{d}\right)$ PW91, and the results of ref. 34 for $\mathrm{Cd}_{4} \mathrm{Se}_{4}$ interacting with a single ligand obtained with PW91, where $n$-BA is $n$-butylamine and $n$-HA is $n$-hexylamine

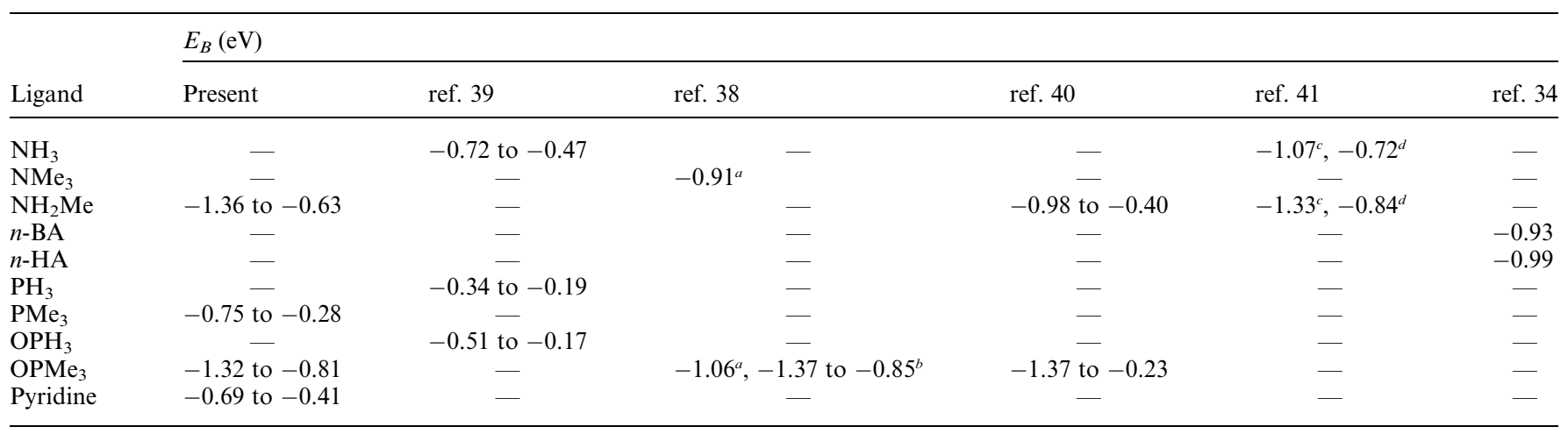

bulk results were obtained with the PW91 functional that is known to underestimate QD-ligand binding energies. ${ }^{57}$ Moreover, the use of $\mathrm{H}$ atoms in place of methyl groups in amine, phosphine and phosphine oxide molecules also results in lower binding energies. ${ }^{30}$ Second, the finite size, shape, and electron confinement are expected to have strong effects on the QD-ligand interactions, resulting in larger absolute values of the binding energies compared to those of CdSe bulk surface. ${ }^{39}$ Results on QD-ligand binding reported in ref. 34 and 38 do not include steric inter-molecular interactions between neighboring ligands on the surface, which were included in the present calculations. As expected, the inter-ligand interactions overall decrease the QD-ligand binding, explaining why our results are somewhat smaller (in absolute values) than those obtained for the same size QD functionalized with a single ligand. ${ }^{38,57}$ Small deviations between the present calculated binding energies and values reported in ref. 40 for the same systems can be explained by differences in methodologies used. Our recent studies ${ }^{57}$ have demonstrated that values of binding energies are extremely method-sensitive, especially to the basis set choice.

In qualitative agreement with all previous calculations, ${ }^{34,38-41}$ phosphine oxides and amines exhibit the strongest binding, on average, to the QD surface. Pyridines and phosphines exhibit weaker interactions with the QD. Reference 39 did find that $\mathrm{PH}_{3}$ has a stronger interaction with the polar, Se terminated face of CdSe than either that of $\mathrm{NH}_{3}$ or $\mathrm{OPH}_{3}$, with $\mathrm{OPH}_{3}$ being essentially nonbinding. Given the experimental evidence ${ }^{16,50}$ pointing to preferential binding of ligands to surface $\mathrm{Cd}$ atoms, we did not explore binding to surface Se atoms in the present study. The magnitude of the binding energies is similar to the activation energy of desorption at room temperature as given by Redhead. ${ }^{63}$ This implies a rather dynamic relationship between QD and ligand.

\subsection{Electronic properties of fully passivated systems: effect of solvent}

In agreement with our previous studies, ${ }^{40}$ all structures demonstrate a well-open energy-gap of $\sim 3 \mathrm{eV}$ (see Fig. 2), which commensurates with the lowest absorption peak $(\sim 2.99 \mathrm{eV})$ experimentally observed and assigned to the $\mathrm{Cd}_{33} \mathrm{Se}_{33}$ cluster. ${ }^{45,47}$ Addition of ligands to the $\mathrm{Cd}_{33} \mathrm{Se}_{33}$ QD leads to a slight decrease in the energy gap of the QD compared to the bare cluster, as judged from the DOS presented in Fig. 2. Such shrinking is more pronounced in the presence of solvent and is likely to originate from polarization effects on the surface of the QD. The solvent media directly affects the surface states of the bare QD, stabilizing occupied orbitals, which results in an increase of the energy gap, compared to those in the gas-phase. The energy gap rises with an increase of the solvent polarity (see Fig. S1 in Supplemental Materials $\dagger$ ), too. However, the ligand capping tends to screen the surface states from the solvent and partially inhibits the solvent's ability to stabilize surface occupied orbitals. Therefore, the increase of the energy gap of the passivated QDs in solvent is smaller then that of the bare QD, leading to the larger difference between the energy gaps of the capped and uncapped QDs in solvent. This difference is more dramatic for the bulkier molecules (e.g., $\mathrm{PMe}_{3}$ and $\mathrm{OPMe}_{3}$ ) having a larger screening effect from the solvent.

Fig. 2 shows a fragment decomposition of the total DOS including orbitals localized on the QD and on the ligands. Specifically, displayed are the DOS corresponding to the QD core (the $\mathrm{Cd}$ and $\mathrm{Se}$ atoms that retain their bulk-like, tetrahedral coordination), the QD surface (the $\mathrm{Cd}$ and $\mathrm{Se}$ atoms not included in the core designation), and the ligands. As a reference, the DOSs for the uncapped QD surface and core atoms are silhouetted in the background by the gray colors. States near the energy gap - including the highest occupied (HOMO) and the lowest unoccupied (LUMO) molecular orbitals - are mostly contributed from the $\mathrm{Cd}$ and Se surface atoms with a small portion from the QD core to the unoccupied orbitals. Solvent stabilizes the surface states while leaving the core states almost unchanged. This brings the QD surface and core states closer in energy, so that the DOS at the edges of the energy gap has more QD core character when solvent is introduced. Thus solvent leads to a stronger delocalization of orbitals over the QD, as illustrated for the LUMOs in Fig. 3 and the HOMOs in Fig. S2 of Supplemental Materials. $\dagger$ As can be seen in Fig. 3, this delocalization is less noticeable with the $\mathrm{PMe}_{3}$ and $\mathrm{OPMe}_{3}$ ligands, again due to the screening of the QD surface states by the ligands. The QD capped with $\mathrm{NH}_{2} \mathrm{Me}$ 


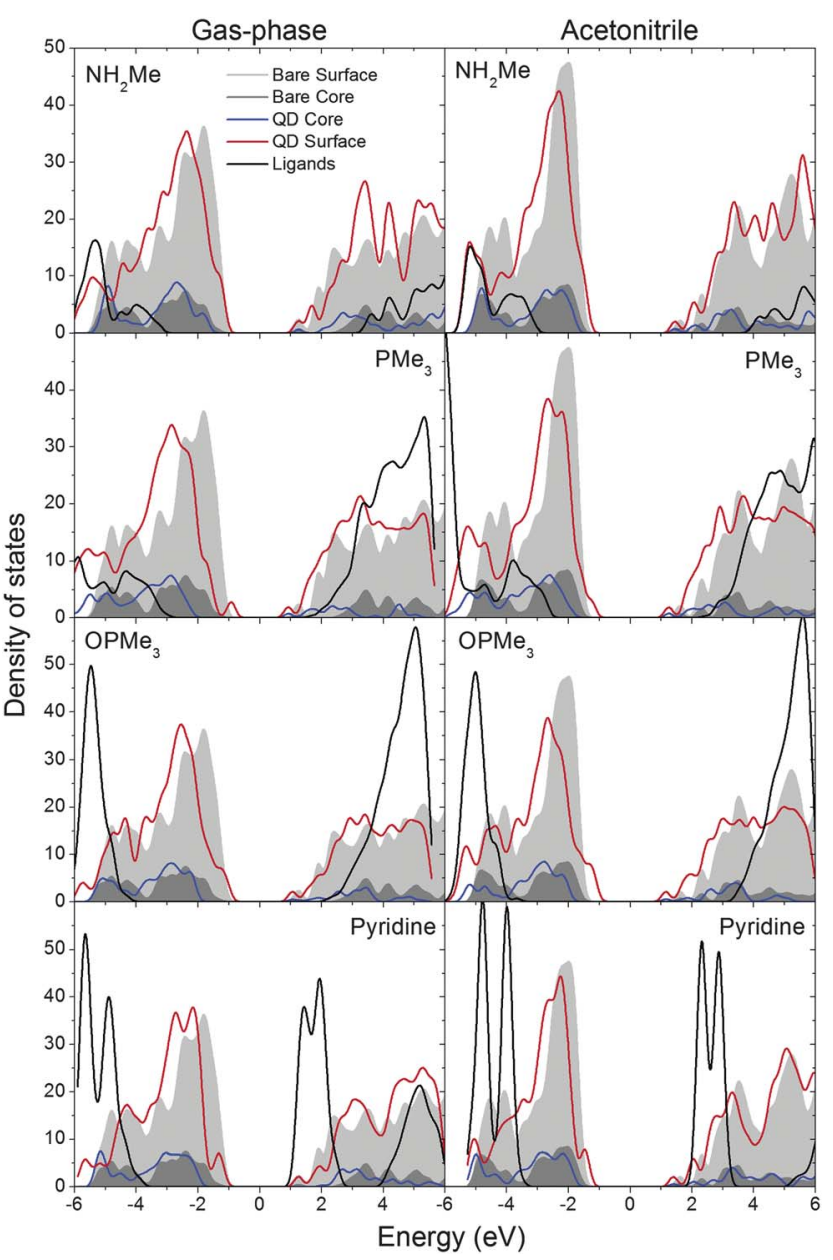

Fig. 2 Fragment analysis of the density of states (DOS) demonstrating states associated with QD-localized (surface and core) and ligand-localized orbitals. The left and right columns show results calculated in the gas-phase and acetonitrile solvent, respectively. The lines correspond to the DOS of the ligated QDs, while the filled curves are the DOS of the uncapped (bare) QD cluster. Except pyridine capped QDs, all ligated structures demonstrate QD character of orbitals close to the edges of the valence and conducted bands. Solvent stabilizes surface orbitals and increases the band gap. Ligand-localized orbitals for pyridine capped QDs are shifted deeper to the conduction band when in solvent.

shows the largest LUMO and HOMO delocalization. Lack of any evidence for localized surface or ligand-associated states close to the energy gap (trap states) exemplify why primary amines and their derivatives are the ligand of choice for achieving high emission quantum yield. ${ }^{15}$

Except pyridine capped QDs, the ligand-localized states appear deep in the valence (VB) and conduction (CB) bands of the passivated $\mathrm{Cd}_{33} \mathrm{Se}_{33}$. In addition, the majority of states associated with ligands have very hybridized character, spreading their orbitals over both ligand and QD atoms. Thus, amines, phosphine and phosphine oxide ligands do not contribute trap states localized on ligands anywhere near the energy gap of the $\mathrm{Cd}_{33} \mathrm{Se}_{33}$ (see Fig. 2). In contrast, pyridinelocalized orbitals are situated just below the CB states of the QD when the system is in the gas-phase, introducing trap states for excited electrons. Solvent, however, shifts pyridine states inside
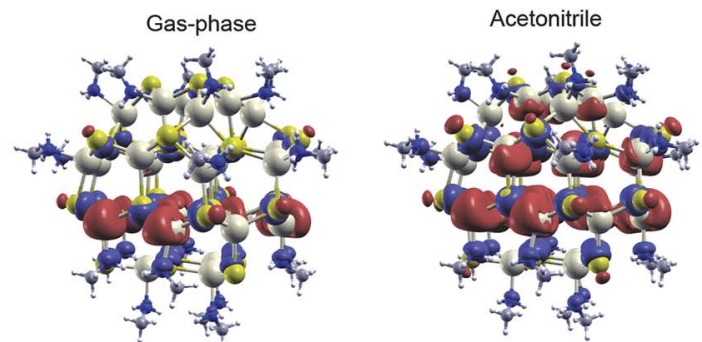

$\mathrm{NH}_{2} \mathrm{Me}$
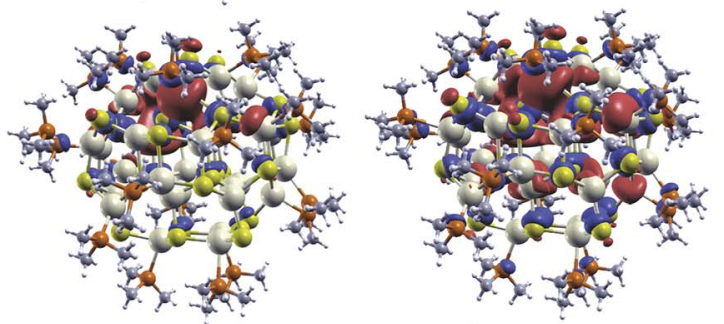

$\mathrm{PMe}_{3}$
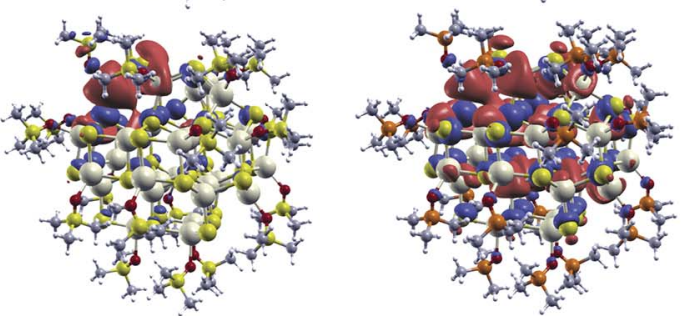

$\mathrm{OPMe}_{3}$
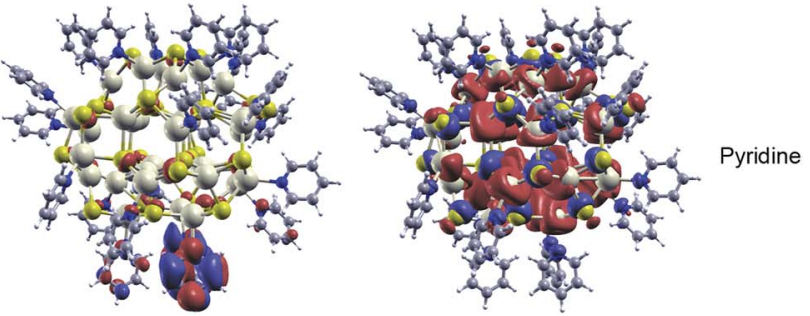

Fig. 3 The lowest unoccupied molecular orbitals (LUMO) of ligated $\mathrm{Cd}_{33} \mathrm{Se}_{33}$ clusters. Orbitals in the left and right columns are calculated in the gas-phase and acetonitrile solvent, respectively. Solvent environment noticeably increases delocalization of the orbital. In the pyridine ligated QD, solvent also shifts pyridine-localized orbital deeper to the conduction band leaving a QD-delocalized orbital as a LUMO.

the $\mathrm{CB}$ of the $\mathrm{Cd}_{33} \mathrm{Se}_{33}$ and hybridizes the orbitals between the ligand and the QD atoms, as can been seen in Fig. 2. The transformation of the LUMO character of the pyridine capped QD is clearly displayed in Fig. 3, where the LUMO changes from being highly localized on a single pyridine ligand in the gasphase, to a state delocalized over the entire QD in solvent.

Pyridine ligands have been reported previously as efficient hole acceptors. ${ }^{14,17,64}$ Although, pyridine-localized occupied orbitals are deep in the VB, our results indicate that the pyridine capped $\mathrm{Cd}_{33} \mathrm{Se}_{33}$ has highly localized surface states at the VB edge (see Fig. S2 in Supplemental Materials $\dagger$ ), which potentially could trap holes. Our calculations suggest that states localized on the pyridines themselves are only capable of trapping highly excited holes, given these states positioning deep inside the VB. Similar surface hole trap states also seem to be present in the cluster capped with $\mathrm{PMe}_{3}$ demonstrating localized QD surface state at the edge of the VB (see Fig. S2 of Supplemental Materialst); however, solvent tends to increase delocalization of these states, 
thus, destabilizing localized trap states and removing them from the energy gap of the QDs.

\subsection{Effect of the loss of a ligand: gas-phase vs. solvent calculations}

Colloidal CdSe QDs are synthesized in the solution phase. Precise control of the QD surface in such dynamic environment is challenging. The magnitude of the calculated binding energies also suggest that the passivation level of the QD in solution near room temperature could be in a state of flux. Therefore, it is necessary to understand the effects incomplete passivation can have on the electronic properties of the QD. This would help to characterize whether experimental measurements signify surface effects or are results intrinsic to the QD itself. To gain insights into these questions, we removed a single ligand from the surface of each of our passivated QDs and recalculated the electronic properties.

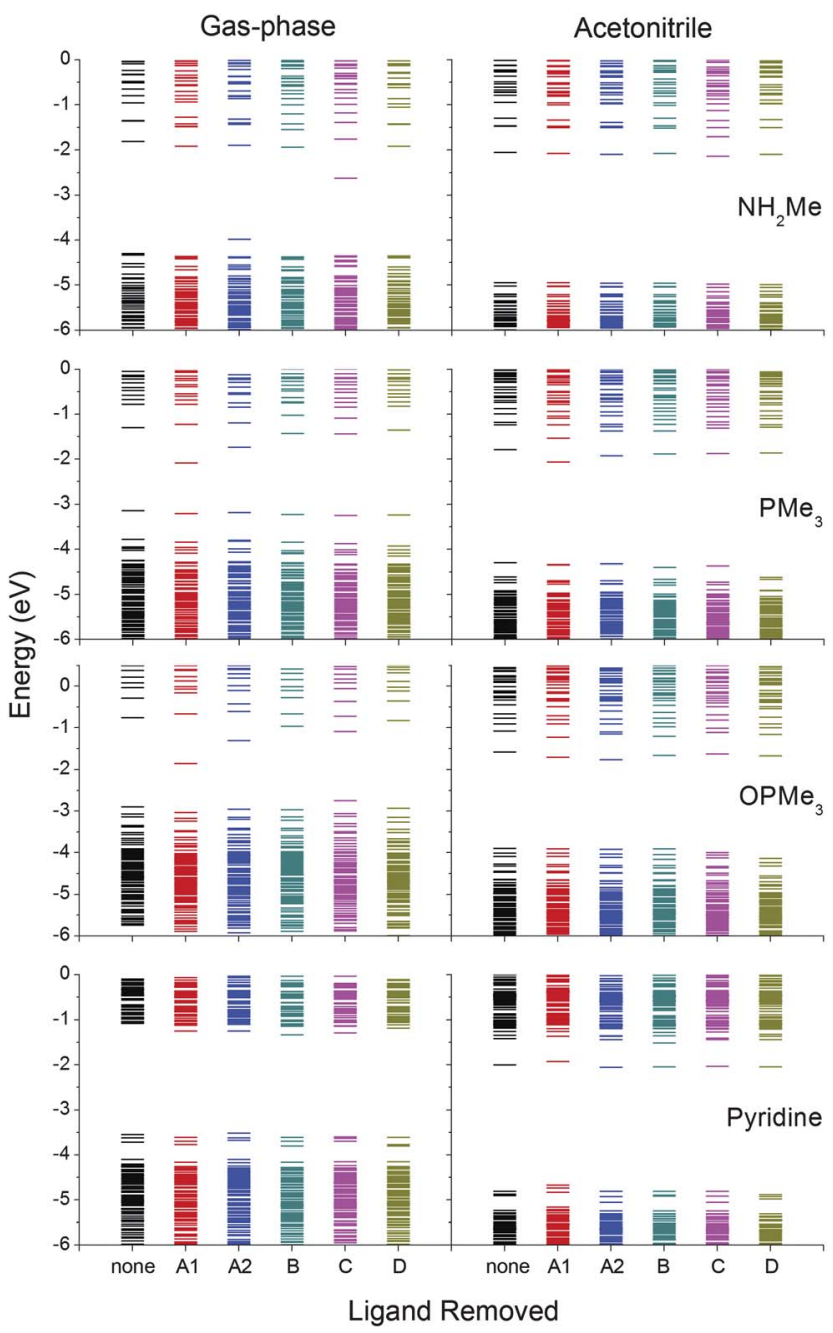

Fig. 4 Kohn-Sham energy level diagram for the fully passivated $\mathrm{Cd}_{33} \mathrm{Se}_{33}$ with 21 ligands and clusters with 20 ligands (i.e. one ligand has been removed from the specific position as indicated underneath the diagram). Positions $\mathrm{A} 1$ and $\mathrm{C}$ are two-coordinated $\mathrm{Cd}$ atoms while the rest are three-coordinated $\mathrm{Cd}$ atoms (see Fig. 1). Solvent stabilizes the energy levels that are disrupted by a ligand loss.
Plotted in Fig. 4 are KS energy level diagrams of the systems under consideration. A single ligand at a time was removed from the surface, with the letters denoting the position on the surface lacking the ligand (see Fig. 1, right panel). Positions A1 and C are two-coordinated $\mathrm{Cd}$ atoms (i.e., bearing two dangling bonds before passivation), while positions $\mathrm{A} 2, \mathrm{~B}$, and $\mathrm{D}$ are threecoordinated $\mathrm{Cd}$ atoms (i.e., having only one dangling bond before passivation). In the gas-phase, appearance of states inside the band gap of the fully passivated QD is mostly observed when ligands are removed from sites A1, A2, or C for all capping ligands (see left column in Fig. 4). Previous studies have shown that ligands generally bind stronger to two-coordinated $\mathrm{Cd}$ atoms on the (1120) facet $^{39}$ and, specifically, to the sites A1, A2 and $\mathrm{C}$ for our particular cluster. ${ }^{40}$ Stronger binding to these sites explains the more pronounced changes seen in the electronic structure of passivated $\mathrm{Cd}_{33} \mathrm{Se}_{33}$ with one ligand removed from these sites as compared to positions B and D.

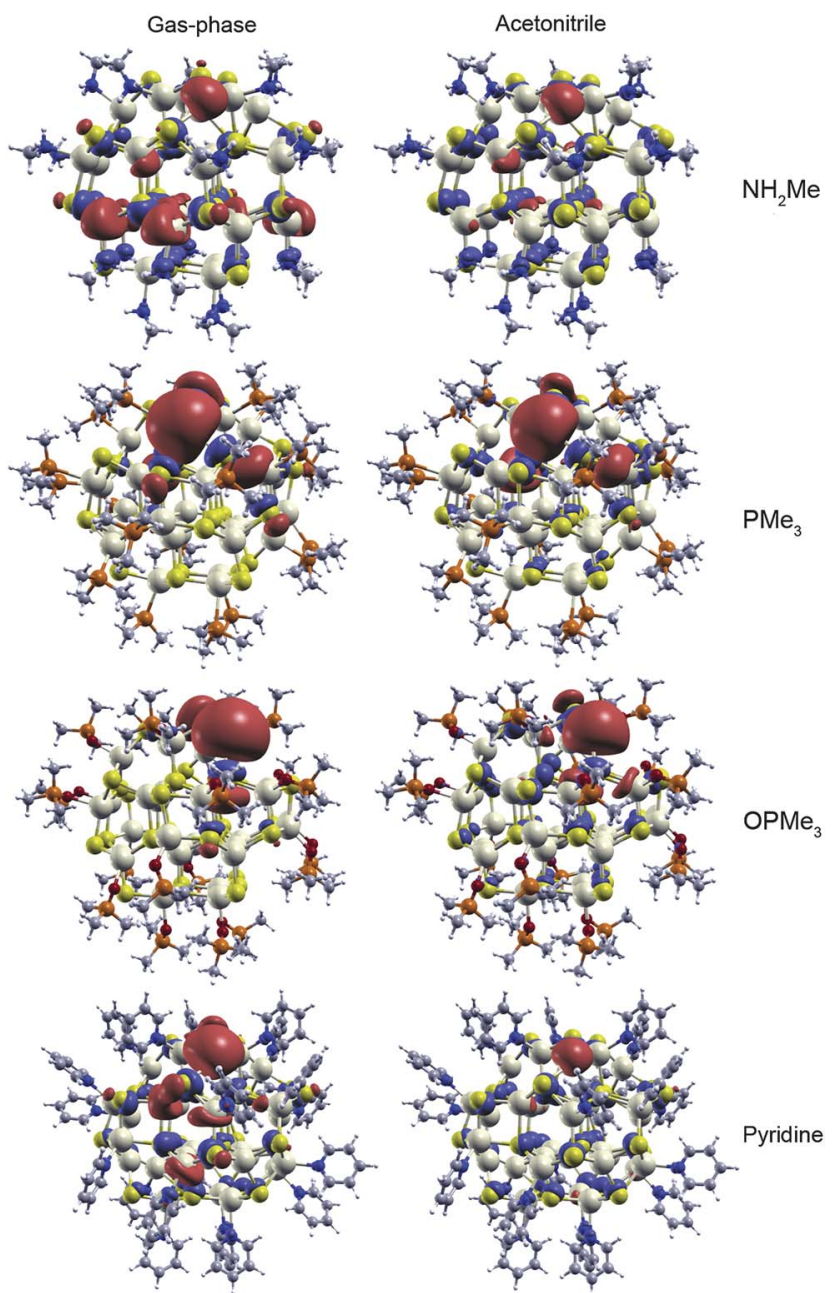

Fig. 5 The lowest unoccupied molecular orbitals (LUMO) of ligated $\mathrm{Cd}_{33} \mathrm{Se}_{33}$ clusters after removal of a single ligand molecule calculated in the gas-phase (left column) and in acetonitrile (right column). In the gasphase, orbitals are localized around the $\mathrm{Cd}$ atom from which a ligand was removed. While solvent delocalizes the orbitals, some degree of localization remains on the respective $\mathrm{Cd}$ atom. 
In general for all gas-phase results shown in Fig. 4 (left column), both LUMO and HOMO (to a lesser degree) enter the gap depending on the removed ligand position, thus introducing states associated with dangling bonds into the energy gap of the QD (see LUMOs in Fig. 5 and HOMOs in Fig. S4 of Supplemental Materials $\dagger$ ). We also note that loss of a ligand slightly perturbs several other orbitals close to the band-edges. The most significant energy level shifts occur after removal of an $\mathrm{OPMe}_{3}$ and $\mathrm{PMe}_{3}$ molecule. Phosphine oxide (phosphine) ligands have the strongest (the weakest) average binding energies of the four ligands we considered, as presented in Table 1. Consequently, ligand-QD binding energy is not the only parameter determining changes in the electronic structure upon detachment of a ligand. Both $\mathrm{PMe}_{3}$ and $\mathrm{OPMe}_{3}$ are relatively bulky molecules, where steric hindrance provides significant interactions between ligands. These inter-ligand interactions likely affect the electronic structure of the system, in addition to the ligand-QD interactions. Similar trends are observed with amine ligands. $\mathrm{NH}_{2} \mathrm{Me}$ has a strong binding energy to the QD surface (comparable to $\mathrm{OPMe}_{3}$, see Table 1), while it is the smallest ligand with small steric inter-ligand interactions. As such, the energy levels of the $\mathrm{NH}_{2} \mathrm{Me}$ capped QD are less perturbed after removal of a ligand than those of the $\mathrm{OPMe}_{3}$ and $\mathrm{PMe}_{3}$ capped QDs. The least amount of modification to the energy levels after removal of a ligand is observed for a pyridine capped QD. This is because pyridine binds relatively weakly to the QD surface and its planar geometry allows for less steric hindrance between ligands. Thus, the effect of removing a ligand on the electronic structure appears to be dependent on both binding capacity of the ligand to the QD and steric inter-ligand interactions.

However, a solvent environment significantly diminishes the ligand-loss effects by screening the binding between the QD and ligands (and, thus, decreasing their binding energies ${ }^{57}$ ), stabilizing the surface states, and opening up the band gap, as discussed above. In fact, in all cases of ligand removal considered, solvent restored the energy levels back to nearly the same configuration as the fully passivated structures (see the right column in Fig. 4). Such rearrangement of the electronic levels originates from the delocalization of orbitals in the presence of solvent, which reduces the local impact of ligand loss. Fig. 5 illustrates this point by showing the LUMO plots of each system with a ligand removed from the A1 position. As expected, detachment of a ligand from the $\mathrm{Cd}_{33} \mathrm{Se}_{33}$ cluster results in the LUMO being localized around the respective 'ligand-free' surface Cd atom (compare Fig. 3 and 5).

Interestingly, LUMOs of QDs capped with $\mathrm{PMe}_{3}$ and $\mathrm{OPMe}_{3}$ calculated in the gas-phase are much more localized at such $\mathrm{Cd}$ atoms, showing s-like character of the unoccupied orbital corresponding to the dangling bonds. It is known that such dangling bonds can be saturated by surface reconstructions. ${ }^{35,65}$ However, the steric hindrance between bulky ligands holds back the surface atoms from moving closer to each other in order to saturate dangling bonds via surface modifications. Thus, the bulky ligands demonstrate the largest localization and dangling bond character due to ligand loss, as compared with those of amine and pyridine capped QDs. When solvent is included in our modeling (see the right column in Fig. 5), the LUMO becomes more delocalized, yet, a considerable portion of the orbital density remains localized on the affected surface $\mathrm{Cd}$ atom.
Consistent with the previous speculations, bulky ligands, such as phosphines and phosphine oxides, screen the effect of solvent on delocalization of surface orbitals leading to more localized orbitals, even in a polar dielectric environment, compared to $\mathrm{NH}_{2} \mathrm{Me}$ and pyridine passivated QDs. In general, our calculations suggest that detachment of one or a few ligands from the QD surface leads to relatively minor changes in the electronic structure and optical spectra of CdSe QDs in polar organic solvent.

\subsection{Analysis of absorption spectrum}

The TDDFT technique has made excited state properties computationally accessible for a broad range of molecular systems. Yet, it is still difficult to obtain anything beyond a few low-lying excited states for large nanostructures such as QDs. In contrast, the Kohn-Sham (KS) single-particle approximation (see eqn (3)) is substantially less computationally expensive than the TDDFT method, allowing application to larger systems and addressing vastly higher excitation energies. ${ }^{40}$ Lacking electronhole correlations though, the KS approach is expected to be less accurate than TDDFT or even not applicable at all, e.g., for some small molecules. To determine whether the errors of a single-particle approximation are small enough to be overlooked for the description of optical properties of QDs, the absorption spectra of ligated $\mathrm{Cd}_{33} \mathrm{Se}_{33}$ have been calculated for both KS and TDDFT approaches.

Fig. 6 compares the KS and TDDFT calculated absorption spectra for all capped QDs in the gas-phase and in acetonitrile, with the spectra for the uncapped QD silhouetted in the background as a reference point. While all absorption features are calculated in the $\mathrm{KS}$ approach in the energy range up to $4 \mathrm{eV}$, the TDDFT results are limited by a much smaller energy window spanned by the first 50 excited-singlet states. In the low-energy region, aside from the systematic blue-shift by $\sim 0.3 \mathrm{eV}$, the profiles of KS spectra are remarkably similar to that of TDDFT spectra. Barring the minor differences in relative peak height and position, the KS spectra capture all essential features of the TDDFT spectra for all ligands. Thus, the KS single-particle approach is sufficiently accurate to qualitatively describe the absorption spectra peaks of QDs. This conclusion agrees with results of a recent theoretical study. ${ }^{66}$ The consistency between KS single-particle picture and electron-hole correlated TDDFT results in the case of QDs can be explained by the dominance of the quantum confinement effects on the optical properties of the QD, compared to excitonic effects. In semiconductor QDs, electron-hole Coulomb energy is much smaller than kinetic energy, ${ }^{67}$ and, therefore, the zeroth-order approximation for photoexcitations as transitions between single-particle orbitals remains valid here. Finally, it should be noted that well established problem of TDDFT in producing spurious low-energy charge transfer states ${ }^{68,69}$ may affect our results as well, where several low-energy optically forbidden states have been calculated, for example, for phosphine passivated QD (see Fig. 6). Consequently, recently-developed range-separated functionals ${ }^{\mathbf{7 0}}$ with large fraction of orbital exchange may be required to address these TDDFT issues.

Having examined the ability of the KS approach to qualitatively reproduce the optical response of QDs, we now focus on 


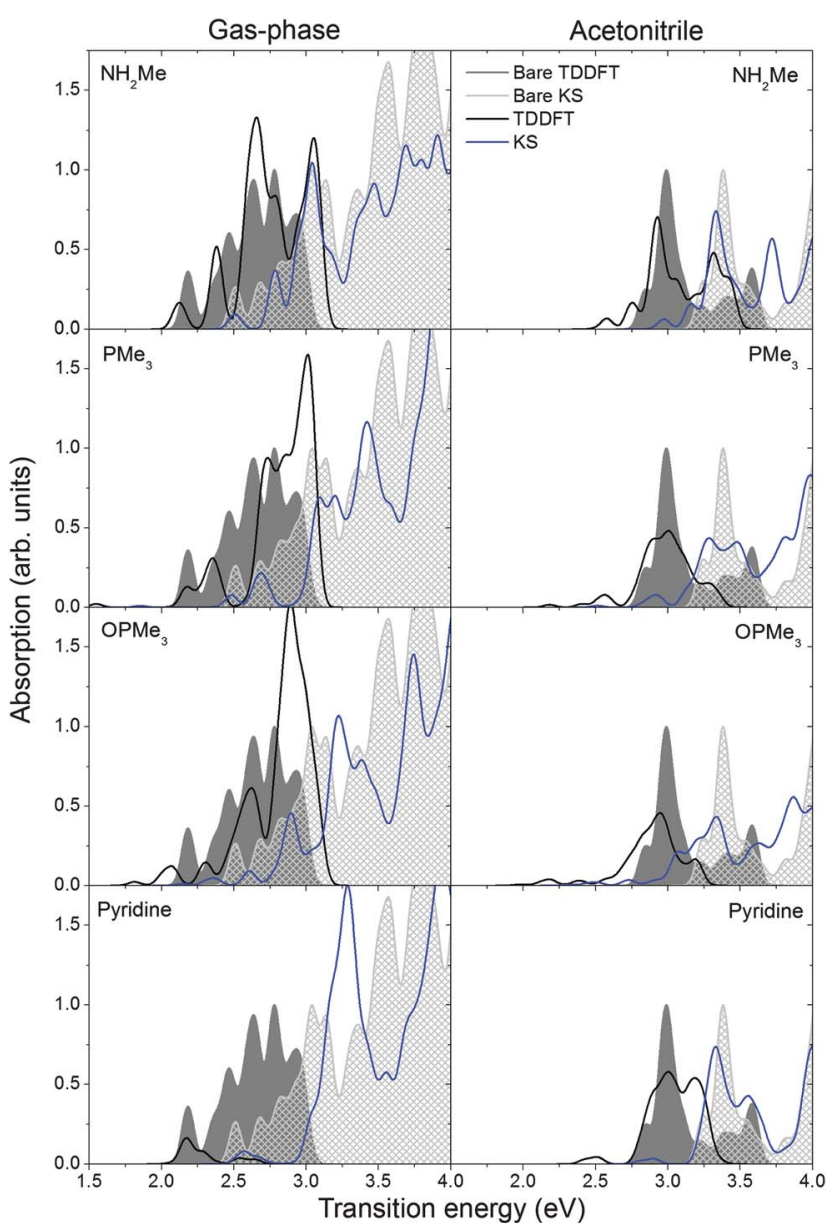

Fig. 6 Calculated optical absorption spectra of ligated quantum dots in the gas-phase (left column) and in acetonitrile solvent (right column). The black and blue lines represent the spectra calculated with TDDFT method and a single-particle KS approach, respectively. The filled curves show the corresponding spectra of the uncapped (bare) QD cluster. All intensities are normalized to that of the TDDFT result for the uncapped QD. Aside from systematic blue-shifts, the KS spectra are in a very good agreement with the respective TDDFT spectra. Solvent introduce noticeable blue shifts of absorption peaks.

the analysis of the absorption spectra. First, we comment on the very small peaks in the TDDFT spectra of the pyridine ligated QD in the gas-phase, compared to those of the bare cluster and QD passivated by other ligands, as presented in Fig. 6 in the left column. We recall that the TDDFT spectrum only includes the first 50 excited states, most of which originate from the electronic transitions from the occupied states localized on the QD to the unoccupied states localized on the pyridine orbitals placed at the edge of the CB of the pyridine capped QD (see DOS in Fig. 2). Indeed, such excitations have extremely low oscillator strengths because of small overlaps between QD-associated initial and ligand-localized final states. This can be seen explicitly by looking at the density of transitions (DOT) which considers all possible single particle transitions from occupied to unoccupied orbitals (Fig. 7). To characterize the nature of transitions, we split the DOT into contributions from different types of transitions such as QD state to QD state or QD state to ligand state. The DOT in Fig. 7 demonstrates that most of the low energy

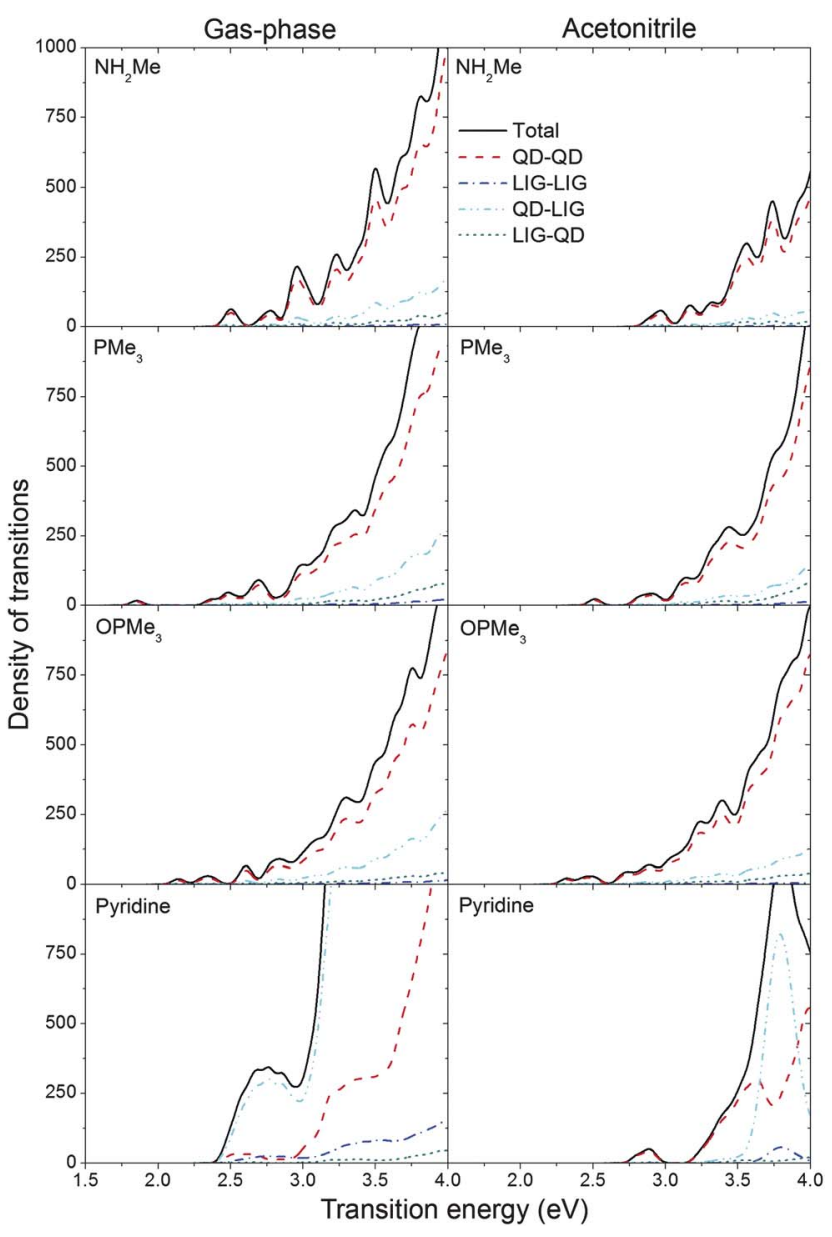

Fig. 7 Calculated density of transitions (DOT) through the band gap of ligated quantum dots in the gas-phase (left column) and in acetonitrile solvent (right column). The DOT includes all possible single-electron KS transitions regardless of their oscillator strength, and is split into contributions from different types of transitions such as QD state to QD state or QD state to ligand state. The majority of transitions at low energies occur between QD orbitals, except for pyridine capped QDs in the gas-phase.

transitions in the ligated $\mathrm{Cd}_{33} \mathrm{Se}_{33}$ occur between QD states, except for pyridine capped QDs in the gas-phase, as also can be confirmed from the DOS in Fig. 2.

Including solvent in the calculation shifts the pyridine states further inside the $\mathrm{CB}$ of the QD resulting in QD to QD transitions appearing at lower energies and enhancing the low-energy absorption (compare Fig. 6 and 7). While the QD to ligand transitions here are 'dark' states with vanishing oscillator strength, which do not contribute to optical absorption, they could play an important role in energy relaxation through electron-phonon coupling with the high-frequency vibrations in the ligands. Moving to general properties of all systems we considered, inclusion of solvent leads to a substantial blue-shift (0.3-0.4 $\mathrm{eV}$ ) of the absorption spectra, which is attributed to the stabilization of the surface states as is seen in the DOS and the KS energy level diagrams (Fig. 2 and 4). With decrease of the solvent dielectric constant, this blue shift lessens, approaching the gasphase results (see Fig. S3 in Supplemental Materials $\dagger$ ). 
Interestingly, the spectra of $\mathrm{PMe}_{3}$ and $\mathrm{OPMe}_{3}$ capped QDs look quite similar to each other with a small red shift of the lowest peaks compared to those of amine and pyridine ligated QDs. Recent experiments have also shown that treatment of QDs with pyridine leads to a slight blue-shift in the optical absorption spectrum. ${ }^{62}$ The intensity of the peaks for the $\mathrm{OPMe}_{3}$ and $\mathrm{PMe}_{3}$ capped QDs is also smaller than for QDs capped by amines and pyridines. These results suggest that photoluminescence (PL) in phosphine and oxide phosphine capped QDs will be slightly red shifted and less efficient as compared to amine ligated QDs. This is corroborated by experimental measurements ${ }^{15,71}$ demonstrating a slight blue shift in PL going from trioctylphosphine oxide (TOPO)/trioctylphosphine (TOP) to amine passivated QDs, as well as an increase in PL quantum yield of CdSe QDs passivated with primary alkylamines. ${ }^{15}$

\section{Conclusions}

In the present study, we analyzed the effects induced by four different commonly used capping ligands on the electronic and optical properties of the $\mathrm{Cd}_{33} \mathrm{Se}_{33}$ cluster. Our DFT calculations demonstrate that amine and phosphine oxide ligands provide stronger binding with the QD surface than phosphines and pyridines. The binding energies of all studied ligands are such that thermal fluctuations at or above room temperature could lead to the detachment of a ligand molecule from the surface.

The random detachment of one of ligands from the QD surface results in a destabilization of the states near the bandedges, with the LUMO being highly localized around the $\mathrm{Cd}$ atom that lost its ligand molecule. We find that the degree to which the electronic structure is perturbed appears related to both the binding energy of the ligand and the degree of steric ligand-ligand interactions. Polar solvent, however, significantly diminishes these effects by reopening the energy gap and delocalizing orbitals, yet it fails to completely remove the localization from the affected surface $\mathrm{Cd}$ atom.

While full passivation of the QD surface by ligands has minimal effect on the size of the HOMO-LUMO gap, as compared to the uncapped but reconstructed cluster, the nature of the states near the band-edges is modified. We observed localized surface states at the VB edge (hole trap states) with phosphine and pyridine ligands, while amines and phosphine oxide ligands provide orbitals well-delocalized over the QD atoms. Importantly, the trap states are found to be localized on the QD surface, rather than on the ligands. The states beyond the band-edges are of a hybridized nature where an orbital is spread over the QD and ligand atoms. These state could enhance nonradiative relaxation within the bands through electron-phonon coupling with the high-frequency vibrations of the ligands.

Consistent with experimental data on the emission quantum yield in amine capped QDs, ${ }^{15}$ our amine passivated QD shows the most orbital delocalization and has the least amount of surface states near the band edges. Phosphine oxide and phosphine capped QDs have slightly more localized HOMO/LUMO orbitals, and exhibit a slight red shift in the lowest optically allowed transitions, compared to amine capped QDs, which also agrees with experimental data. ${ }^{71}$ The higher degree of localization of the near band-edge states could hinder radiative recombination.
Our calculations demonstrate that a dielectric environment (solvent) has important effects and needs to be incorporated into quantum-chemical calculations for the proper description of the electronic structure and optical properties of ligated QDs. Including solvent in the calculations results in an increase of the HOMO-LUMO gap by stabilizing the surface states. Thus, surface-trap and ligand-localized states are substantially eliminated by the polar solvent. Polar solvent also blue-shifts $(0.3-0.4$ $\mathrm{eV}$ in acetonitrile) computed absorption spectra: The larger the dielectric constant of the solvent, the larger the observed blueshift. This is rationalized by the polar character of both QD and ligand structures, whose dipole moments are screened by the solvent environment.

We also found that for the low-energy absorption spectra, the KS single-particle approach agrees well with the results obtained with TDDFT methodology. Similar results were recently reported as well. ${ }^{66}$ All major features of the spectra are captured in the KS approach with the only difference being a systematic blueshift of the KS spectra relative to the TDDFT spectra. Notably that the amount of this blue-shift strongly depends on the fraction of the orbital exchange included in the underlying density functional kernel. ${ }^{72}$ Such qualitative level of agreement between KS and TDDFT methods, ${ }^{66}$ reflects that the kinetic energy is significantly larger than the electron-hole Coulomb interaction in these systems due to quantum confinement.

Overall, the analysis presented in this study should benefit to both future theoretical and experimental studies. We have demonstrated what details are necessary and what approximations can be made in the calculation of QD properties. In order to fully exploit the unique properties of QDs for use in the wide variety of proposed applications, control over the surface and passivation characteristics of the system must be attained. This can only be reliably achieved with a detailed understanding of the ligand-QD interactions and dynamics.

\section{Acknowledgements}

SAF, AMC and ST acknowledge support from the Center for Advanced Solar Photophysics, an Energy Frontier Research Center funded by the U.S. Department of Energy (DOE), Office of Science, Office of Basic Energy Sciences (BES). SK acknowledges financial support from ND EPSCoR and NSF grant no. EPS-0814442 and DOE start up grant no. DE-FG3608GO88160. We also acknowledge support of Center for Integrated Nanotechnology (CINT) and Center for Nonlinear Studies (CNLS) at Los Alamos National Laboratory. Los Alamos National Laboratory is operated by Los Alamos National Security, LLC, for the National Nuclear Security Administration of the U.S. Department of Energy under contract DE-AC5206NA25396.

\section{References}

1 L. E. Brus, J. Chem. Phys., 1984, 80, 4403-4409.

2 A. I. Ekimov, A. L. Efros and A. A. Onushchenko, Solid State Commun., 1985, 56, 921-924.

3 A. P. Alivisatos, Science, 1996, 271, 933-937.

4 M. Dahan, S. Levi, C. Luccardini, P. Rostaing, B. Riveau and A. Triller, Science, 2003, 302, 442-445.

5 J. Gorman, D. G. Hasko and D. A. Williams, Phys. Rev. Lett., 2005, 95, 090502 . 
6 V. I. Klimov, A. A. Mikhailovsky, S. Xu, A. Malko, J. A. Hollingsworth, C. A. Leatherdale, H. J. Eisler and M. G. Bawendi, Science, 2000, 290, 314-317.

7 R. D. Schaller and V. I. Klimov, Phys. Rev. Lett., 2004, 92, 186601.

8 C. B. Murray, D. J. Norris and M. G. Bawendi, J. Am. Chem. Soc., 1993, 115, 8706-8715.

9 L. H. Qu, A. Peng and X. G. Peng, Nano Lett., 2001, 1, 333-337.

10 D. V. Talapin, J.-S. Lee, M. V. Kovalenko and E. V. Shevchenko, Chem. Rev., 2010, 110, 389-458.

11 D. J. Norris and M. G. Bawendi, Phys. Rev. B: Condens. Matter, 1996, 53, 16338-16346.

12 J. Jasieniak, L. Smith, J. Van Embden, P. Mulvaney and M. Califano, J. Phys. Chem. C, 2009, 113, 19468-19474.

13 J. Jasieniak, M. Califano and S. Watkins, ACS Nano, 2011, 5, 5888 5902.

14 P. Guyot-Sionnest, B. Wehrenberg and D. Yu, J. Chem. Phys., 2005, 123, 074709 .

15 D. V. Talapin, A. L. Rogach, A. Kornowski, M. Haase and H. Weller, Nano Lett., 2001, 1, 207-211.

16 I.-S. Liu, H.-H. Lo, C.-T. Chien, Y.-Y. Lin, C.-W. Chen, Y.-F. Chen, W.-F. Su and S.-C. Liou, J. Mater. Chem., 2008, 18, 675-682.

17 P. Guyot-Sionnest, M. Shim, C. Matranga and M. Hines, Phys. Rev. B: Condens. Matter, 1999, 60, R2181-R2184.

18 Y. Chen, J. Vela, H. Htoon, J. L. Casson, D. J. Werder, D. A. Bussian, V. I. Klimov and J. A. Hollingsworth, J. Am. Chem. Soc., 2008, 130(15), 5026-5027.

19 M. C. Beard, A. G. Midgett, M. Law, O. E. Semonin, R. J. Ellingson and A. J. Nozik, Nano Lett., 2009, 9, 836-845.

20 S. Pokrant and K. B. Whaley, Eur. Phys. J. D, 1999, 6, 255-267.

21 L. W. Wang and A. Zunger, Phys. Rev. B: Condens. Matter, 1996, 53, 9579-9582.

22 F. A. Reboredo and A. Zunger, Phys. Rev. B: Condens. Matter, 2001, 63, 235314

23 E. Rabani, J. Phys. Chem. C, 2009, 113, 1493-1497.

24 P. Schapotschnikow, B. Hommersom and T. Vlugt, J. Phys. Chem., 2001, 115, 12690-12698.

25 K. A. Nguyen, P. N. Day and R. Pachter, J. Phys. Chem. C, 2010, 114, 16197-16209.

26 G. M. Dalpian, M. L. Tiago, M. L. del Puerto and J. R. Chelikowsky, Nano Lett., 2006, 6, 501-504.

27 M. L. del Puerto, M. L. Tiago and J. R. Chelikowsky, Phys. Rev. Lett., 2006, 97, 096401.

28 E. Badaeva, Y. Feng, D. R. Gamelin and X. Li, New J. Phys., 2008, 10, 055013 .

29 M. Green, J. Mater. Chem., 2010, 20, 5797-5809.

30 P. Yang, S. Tretiak, A. E. Masunov and S. Ivanov, J. Chem. Phys., 2008, 129, 074709 .

31 T. M. Inerbaev, A. E. Masunov, S. I. Khondaker, A. Dobrinescu, A.-V. Plamada and Y. Kawazoe, J. Chem. Phys., 2009, 131, 044106.

32 S.-Y. Chung, S. Lee, C. Liu and D. Neuhauser, J. Phys. Chem. B, 2009, 113, 292-301.

33 C. M. Isborn, S. V. Kilina, X. Li and O. V. Prezhdo, J. Phys. Chem. C, 2008, 112(47), 18291-18294.

34 H.-L. Chou, C.-H. Tseng, C. Pillai, B.-J. Hwang and L.-Y. Chen, Nanoscale, 2010, 2, 2679-2684

35 A. Puzder, A. J. Williamson, F. Gygi and G. Galli, Phys. Rev. Lett., 2004, 92, 217401.

36 H. Kamisaka, S. V. Kilina, K. Yamashita and O. V. Prezhdo, J. Phys. Chem. C, 2008, 112, 7800-7808.

37 S. V. Kilina, D. S. Kilin and O. V. Prezhdo, ACS Nano, 2009, 3, 93 99.

38 A. Puzder, A. J. Williamson, N. Zaitseva, G. Galli, L. Manna and A. P. Alivisatos, Nano Lett., 2004, 4, 2361-2365.

39 J. Y. Rempel, B. L. Trout, M. G. Bawendi and K. F. Jensen, J. Phys. Chem. B, 2006, 110, 18007-18016.

40 S. Kilina, S. Ivanov and S. Tretiak, J. Am. Chem. Soc., 2009, 131, $7717-7726$.

41 M. Del Ben, R. W. A. Havenith, R. Broer and M. Stener, J. Phys Chem. C, 2011, 115, 16782-16796.

42 C. L. Wang, S. H. Xu, L. H. Ye, W. Lei and Y. P. Cui, Struct. Chem., 2010, 21, 1215-1219.

43 S. H. Xu, C. L. Wang and Y. P. Cui, Struct. Chem., 2010, 21, 519-525.

44 S. H. Xu, C. L. Wang and Y. P. Cui, J. Mol. Model., 2009, 16, 469 473 .
45 A. Kasuya, R. Sivamohan, Y. A. Barnakov, I. M. Dmitruk, T. Nirasawa, V. R. Romanyuk, V. Kumar, S. V. Mamykin, K. Tohji, B. Jeyadevan, K. Shinoda, T. Kudo, O. Terasaki, Z. Liu, R. V. Belosludov, V. Sundararajan and Y. Kawazoe, Nat. Mater., 2004, 3, 99-102.

46 V. Dzhagan, N. Melnik, O. Rayevska, G. Grozdyuk, V. Strelchuk, O. Plyashechnik, S. Kuchmii and M. Valakh, Phys. Status Solidi $R R L, 2011,5,250-252$

47 S. Kudera, M. Zanella, C. Giannini, A. Rizzo, Y. Li, G. Gigli, R. Cingolani, G. Ciccarella, W. Spahl, W. J. Parak and L. Manna, Adv. Mater., 2007, 19, 548.

48 O. Voznyy, J. Phys. Chem. C, 2011, 115, 15927-15932.

49 Y. Feng, E. Badaeva, D. R. Gamelin and X. Li, J. Phys. Chem. Lett., 2010, 1, 1927-1931.

50 L. R. Becerra, C. B. Murray, R. G. Griffin and M. G. Bawendi, J. Chem. Phys., 1994, 100, 3297-3300.

51 J. T. Kopping and T. E. Patten, J. Am. Chem. Soc., 2008, 130, 56895698.

52 J. S. Owen, J. Park, P. Trudeau and A. P. Alivisatos, J. Am. Chem. Soc., 2008, 130, 12279-12281.

53 A. J. Morris-Cohen, M. T. Frederick, G. D. Lilly, E. A. McArthur and E. A. Weiss, J. Phys. Chem. Lett., 2010, 1, 1078-1081.

54 A. Wolcott, R. C. Fitzmorris, O. Muzaffery and J. Z. Zhang, Chem. Mater., 2010, 22, 2814-2821.

55 M. J. Frisch; G. W. Trucks; H. B. Schlegel; G. E. Scuseria; M. A. Robb; J. R. Cheeseman; J. A. Montgomery, Jr; T. Vreven; K. N. Kudin; J. C. Burant; J. M. Millam; S. S. Iyengar; J. Tomasi; V. Barone; B. Mennucci; M. Cossi; G. Scalmani; N. Rega; G. A. Petersson; H. Nakatsuji; M. Hada; M. Ehara; K. Toyota; R. Fukuda; J. Hasegawa; M. Ishida; T. Nakajima; Y. Honda; O. Kitao; H. Nakai; M. Klene; X. Li; J. E. Knox; H. P. Hratchian; J. B. Cross; V. Bakken; C. Adamo; J. Jaramillo; R. Gomperts; R. E. Stratmann; O. Yazyev; A. J. Austin; R. Cammi; C. Pomelli; J. W. Ochterski; P. Y. Ayala; K. Morokuma; G. A. Voth; P. Salvador; J. J. Dannenberg; V. G. Zakrzewski; S. Dapprich; A. D. Daniels; M. C. Strain; O. Farkas; D. K. Malick; A. D. Rabuck; K. Raghavachari; J. B. Foresman; J. V. Ortiz; Q. Cui; A. G. Baboul; S. Clifford; J. Cioslowski; B. B. Stefanov; G. Liu; A. Liashenko; P. Piskorz; I. Komaromi; R. L. Martin; D. J. Fox; T. Keit Gaussian 03, Revision D.02.

56 M. J. Frisch; G. W. Trucks; H. B. Schlegel; G. E. Scuseria; M. A. Robb; J. R. Cheeseman; G. Scalmani; V. Barone; B. Mennucci; G. A. Petersson; H. Nakatsuji; M. Caricato; X. Li; H. P. Hratchian; A. F. Izmaylov; J. Bloino; G. Zheng; J. L. Sonnenberg; M. Hada; M. Ehara; K. Toyota; R. Fukuda; J. Hasegawa; M. Ishida; T. Nakajima; Y. Honda; O. Kitao; H. Nakai; T. Vreven; J. A. Montgomery, Jr; J. E. Peralta; F. Ogliaro; M. Bearpark; J. J. Heyd; E. Brothers; K. N. Kudin; V. N. Staroverov; R. Kobayashi; J. Normand; K. Raghavachari; A. Rendell; J. C. Burant; S. S. Iyengar; J. Tomasi; M. Cossi; N. Rega; J. M. Millam; M. Klene; J. E. Knox; J. B. Cross; V. Bakken; C. Adamo; J. Jaramillo; R. Gomperts; R. E. Stratmann; O. Yazyev; A. J. Austin; R. Cammi; C. Pomelli; J. W. Ochterski; R. L. Martin; K. Morokuma; V. G. Zakrzewski; G. A. Voth; P. Salvador; J. J. Dannenberg; S. Dapprich; A. D. Daniels; O. Farkas; J. B. Foresman; J. V. Ortiz, Gaussian 09 Revision A.1.

57 V. V. Albert, S. A. Ivanov, S. Tretiak and S. V. Kilina, J. Phys. Chem. C, 2011, 115, 15793-15800.

58 Z. Ding, B. M. Quinn, S. K. Haram, L. E. Pell, B. A. Korgel and A. J. Bard, Science, 2002, 296, 1293-1297.

59 N. M. Dimitrijevic and P. V. Kamat, J. Phys. Chem., 1987, 91, 20962099.

60 A. Y. Koposov, T. Cardolaccia, V. Albert, E. Badaeva, S. Kilina, T. J. Meyer, S. Tertiak and M. Sykora, Langmuir, 2011, 27, 83778383.

61 J. Tomasi, B. Mennucci and R. Cammi, Chem. Rev., 2005, 105, 2999_ 3093.

62 V. Dzhagan, I. Lokteva, C. Himcinschi, J. Kolny-Olesiak, M. Y. Valakh, S. Schulze and D. R. T. Zahn, J. Appl. Phys., 2011, 109, 084334

63 P. A. Redhead, Vacuum, 1962, 12, 203-211.

64 V. I. Klimov, A. A. Mikhailovsky, D. W. McBranch, C. A. Leatherdale and M. G. Bawendi, Phys. Rev. B: Condens. Matter, 2000, 61, 13349-13352. 
65 M. Yu, G. W. Fernando, R. Li, F. Papadimitrakopoulos, N. Shi and R. Ramprasad, Appl. Phys. Lett., 2006, 88, 231910.

66 S. A. Fischer, B. F. Habenicht, A. B. Madrid, W. R. Duncan and O. V. Prezhdo, J. Chem. Phys., 2011, 134(2), 024102.

67 A. I. Ekimov, F. Hache, M. C. Schanneklein, D. Ricard, C. Flytzanis, I. A. Kudryavtsev, T. V. Yazeva, A. V. Rodina and A. L. Efros, $J$. Opt. Soc. Am. B, 1993, 10, 100-107.

68 A. Dreuw and M. Head-Gordon, J. Am. Chem. Soc., 2004, 126(12), 4007-4016.
69 R. J. Magyar and S. Tretiak, J. Chem. Theory Comput., 2007, 3(3), 976-987.

70 K. A. Nguyen, P. N. Day and R. Pachter, J. Chem. Phys., 2011, 135 (7), 074109.

71 D. V. Talapin, A. L. Rogach, I. Mekis, S. Haubold, A. Kornowski, M. Haase and H. Weller, Colloids Surf., A, 2002, 202, $145-154$.

72 S. Tretiak, K. Igumenshchev and V. Chernyak, Phys. Rev. B: Condens. Matter Mater. Phys., 2005, 71(3), 33201. 